\title{
A 3D-PRINTED 1 MG LEGGED MICROROBOT RUNNING AT 15 BODY LENGTHS PER SECOND
}

\author{
Ryan St. Pierre ${ }^{1^{*}}$, Walker Gosrich ${ }^{2}$, and Sarah Bergbreiter ${ }^{1}$ \\ ${ }^{1}$ Department of Mechanical Engineering and the Institute for Systems Research, University of Maryland, \\ College Park, Maryland, USA \\ ${ }^{2}$ Department of Mechanical and Aerospace Engineering, The State University of New York at Buffalo, \\ Buffalo, New York, USA
}

\begin{abstract}
This work presents an experimental platform for studying the locomotion of small-scale $(<100 \mathrm{mg})$ legged microrobots. Robot chassis were fabricated with microscale $3 \mathrm{D}$ printing and embedded permanent magnets provide actuation. The design integrates a full rotational friction bearing in the hip joint, capable of actuation up to $150 \mathrm{~Hz}$ with no visible signs of wear after rotating at $100 \mathrm{~Hz}$ for over 1,000,000 cycles. The robot presented in this work weighs 1 $\mathrm{mg}$ and is observed running at speeds up to $37.3 \mathrm{~mm} / \mathrm{s}$ (14.9 body lengths per second) providing initial insights on the dynamics of legged locomotion at ant-scales.
\end{abstract}

\section{INTRODUCTION}

Empirical biological scaling laws show an increase in relative velocity (body length per second) with decreasing body mass [1]. However, as robots are scaled down, their relative speeds pale in comparison to biological counterparts. An understanding of the dynamics of legged locomotion at insect scales is needed to inform design and control of microrobots. Large-scale robots, such as RHex $(7 \mathrm{~kg})$ [2], provide a platform for understanding both the morphology and control of legged locomotion. The large size of RHex enables versatility and flexibility within the platform, allowing design components, such as legs, to be changed easily. By incorporating microscale $3 \mathrm{D}$ printing with magnetic actuation, this work provides a versatile platform to study legged locomotion of robots with a mass over 1,000,000 times smaller than RHex. The final robot tested in this work is shown in Figure 1.

Making ant-scale microrobotics, specifically robots less than $100 \mathrm{mg}$ in body mass, is an impressive feat in itself. One of the more successful microrobots, an $80 \mathrm{mg}$ thermally actuated microrobot, presented in [3] used thermal actuation to raise and lower legs. However, the stride length and stride frequency of this robot was limited, showing top speeds up to $6 \mathrm{~mm} / \mathrm{s}(0.8$ body lengths per second). The $10 \mathrm{mg}$ solar powered microrobot presented in [4] incorporated actuation, control, and power to achieve a robot capable of autonomous movement, although not appreciable forward propulsion. More recently, the walking robot $(18 \mathrm{mg})$ presented in [5] incorporated electrostatic actuators to generate foot paths in vertical and horizontal directions and demonstrated speeds up to $0.7 \mathrm{~mm} / \mathrm{s} \quad(0.15$ body lengths per second).

These same microrobots can serve as physical models for understanding milligram-scale legged locomotion. Legged robots with lead zirconate titanate (PZT) were developed to investigate the dynamics of contact interactions in microrobotic legs [6], with more recent, similarly structured, legged microrobots weighing 4.4 $\mathrm{g}$ and $379 \mathrm{mg}$ demonstrating walking at 0.06 body lengths per second and 0.1 body lengths per second, respectively [7]. The 25 $\mathrm{mg}$ robot fabricated in [8] incorporated compliant materials to create a microrobot with passively articulated legs. By taking advantage of the passive degrees of freedom and incorporating magnetic actuation this robot achieved top speeds of 5 body lengths per second.

Similar to the experimental platform presented in [9], this work takes advantage of $3 \mathrm{D}$ printing to create a chassis for a legged robot. By incorporating permanent magnets as a form of wireless actuation the mechanical aspects of legged locomotion can be studied. Additionally, magnetic actuation provides insights into the torque requirements as well as the mechanical power required to achieve motion.

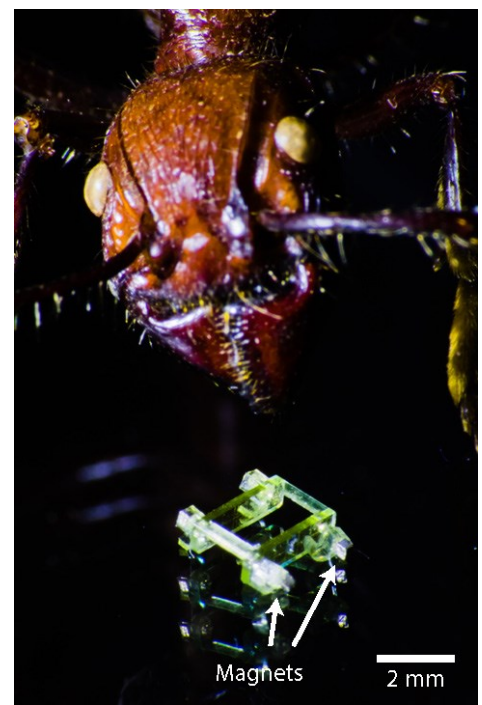

Figure 1: Fabricated $1 \mathrm{mg}, 2.5 \mathrm{~mm} \times 1.6 \mathrm{~mm} \times 0.7 \mathrm{~mm}$ quadrupedal microrobot pictured with a desiccated bullet ant head (Paraponera clavata).

\section{ROBOT DESIGN AND FABRICATION}

The robot design is similar to RHex [2] and the quadrupedal robots presented in [9]. Each leg of the robot is shaped like a "C", providing a rolling contact with the ground. A friction bearing at each hip enables a full rotary motion of the leg from the torque on an embedded permanent magnet.

The microrobots were fabricated using direct laser writing for $3 \mathrm{D}$ printing at the microscale. The process is described in Figure 2. A sacrificial layer of $20 \%$ dextran (Dextran 70 Sigma-Aldrich) was spin-coated on to an ITO-coated glass slide (Nanoscribe) at 1,500 rpm for 1 minute (Figure 2A). The microrobot structure is patterned using dip-in laser lithography (DiLL) with a Nanoscribe Photonic Professional GT using the 25X objective (Carl Zeiss Microscopy) and a negative tone photoresist (IP-S Nanoscribe). After printing, the microrobots were developed in propylene glycol monomethyl ether acetate (PGMEA) for 2 hours. The slide was removed from the PGMEA bath and rinsed with isopropyl alcohol (IPA) to wash away the PGMEA and excess photoresist. The microrobots were released from the sacrificial layer by dissolving the dextran with deionized (DI) water. The microrobots were then 
placed in a bath of IPA and sonicated overnight in a heated bath (3510 MTH Branson) to remove any excess photoresist in the rotary joints of the robot. Once fully cleaned, $250 \mu \mathrm{m}$ cube magnets (C0005-10, SuperMagnetMan) were manually placed in the hip joints and secured with cyanoacrylate (Loctite 401). The final fabricated robot is shown in Figure 1. The orientation of the dipoles of the embedded magnets mechanically programs the gait for the microrobot. In this work, the dipoles were aligned in the same direction, creating a pronking (4-legged hopping) gait.

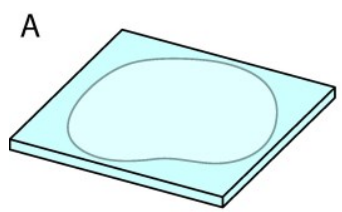

ITO coated slide with a dextran sacrificial layer

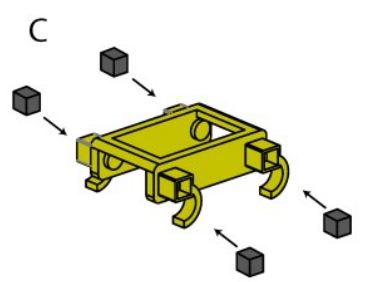

Magnets are manually inserted and secured with glue

Figure 2: Fabrication process for direct laser writing and embedded permanent magnets for actuation.

\section{EXPERIMENTAL SETUP}

Robots were actuated in a custom-made two-axis Helmholtz coil system, used to generate a two-dimensional rotating magnetic field, providing a torque on the embedded magnets and actuating the legs. A schematic of the interaction is shown in Figure 3. The coil brackets are 3D printed (UPrint Plus SE Stratasys) and wrapped with 22 gauge enameled magnet wire. The vertical coil pair has a nominal diameter of $120 \mathrm{~mm}$ and 60 turns of wrapping, while the horizontal pair has a nominal diameter of $160 \mathrm{~mm}$ and 50 turns of wrapping. To create a rotating magnetic field, each channel of a 2-channel arbitrary function generator (AFG3022C Tektronix) was connected to a current amplifier (TS200-0A Accel Instruments). The output of each channel of the function generator is set to balance the coils, creating a symmetric rotating field with a magnitude of $0.78 \mathrm{mT}$. The dipole of the $250 \mu \mathrm{m}$ cube magnets was estimated from the volume and material of the magnets as 17.8 A-mm ${ }^{2}$. Using the point-dipole model in [10], the maximum applied torque is calculated as

$$
\tau_{\max }=\mu_{0} h m_{r}
$$

Where $\mu_{0}$ is the is the magnetic permeability, $\mu_{0}=4 \pi \times 10^{-7} \mathrm{~N} / \mathrm{A}^{2}$ (in vacuum), $h$ is the magnetic field from the coil, and $m_{r}$ is the magnetic dipole of the embedded permanent magnets. The maximum applied torque was calculated as $13.8 \mu \mathrm{N}-\mathrm{mm}$.

To track the motion of the robot, the robot was filmed in the sagittal plane with a high-speed camera (Fastcam UX100) at 1,000 fps equipped with a macro lens (Zeiss Makro-Planar T* $2 / 100 \mathrm{~mm}$ ZF.2 Macro Lens). The field of view of the macro lens was approximately $19 \mathrm{~mm}$ wide, accommodating 7.5 body lengths of travel. The motion of the robot was tracked using markerless tracking software (TEMA Image Systems).

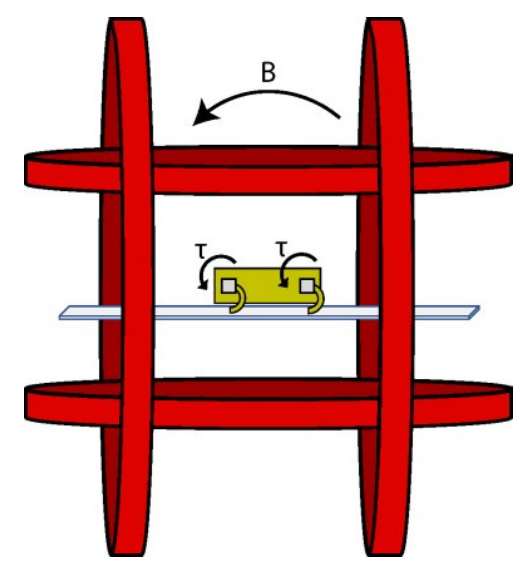

Figure 3: The microrobot is actuated in a rotating magnetic field (B), resulting in a torque applied to each of the embedded magnets in the hip joints. The vertical and horizontal components of the magnetic field are generated from each coil pair.

\section{CHARACTERIZATION OF 3D PRINTED ROTATIONAL BEARINGS}

Fully rotational microdevices are limited in their performance by surface forces, such as friction, while solid-solid interfaces in rotating parts often suffer from wear, further decreasing performance. To minimize the effect of friction in rotary devices, contact needs to be limited by either micro-ball bearings [11], hydro-dynamic bearings [12], air bearings created through magnetic or electrostatic suspension $[13,14]$, or a liquid film $[15,16]$. While most of these strategies require complex manufacturing processes with tight tolerances, the bearings used in this work were printed monolithically, requiring only a single fabrication step. A solid model and photograph of the bearing is shown in Figure 5.
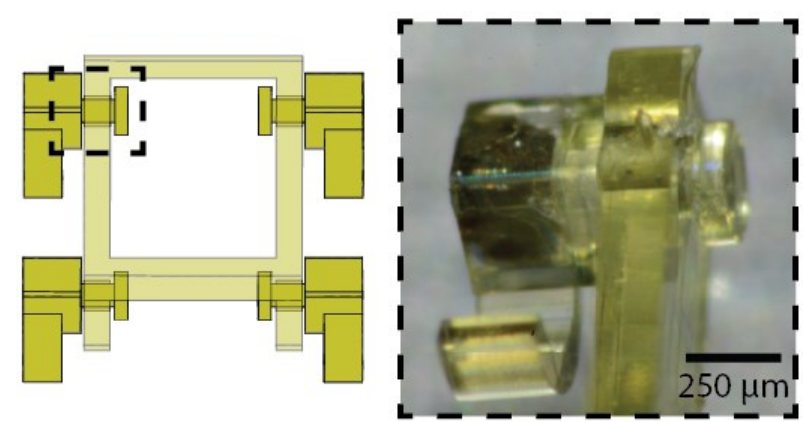

Figure 4: (left) CAD illustration showing the embedded legs within the (transparent) body to create a monolithic bearing. (Right) Photograph of printed component.

\section{Startup torque}

The startup torque is the minimum torque applied for the leg to start moving and overcome static friction within the joint. The startup torque was found by positioning the leg both downward and upward, to compensate for any gravitational effects, and energizing the horizontal coil pair to apply a maximal torque to the leg. The coils are powered by a $420 \mathrm{~W}$ power supply (Sorensen XPF60-20 DPF) slowly increasing the voltage until the leg moves and the corresponding current is recorded. The applied voltage and 
current to the coil is $1.63 \pm 0.02 \mathrm{~V}$ and $0.55 \pm 0.00 \mathrm{~A}$, resulting in a field of $0.31 \mathrm{mT}$, and a calculated start up torque of $5.47 \mu \mathrm{N}-\mathrm{mm}$.

\section{Actuation response}

The robot was suspended without ground contact and actuated from $10 \mathrm{~Hz}$ to $180 \mathrm{~Hz}$ in $10 \mathrm{~Hz}$ increments. High-speed video of the leg rotating was recorded at 2,500 fps (Fastcam UX100) and processed in markerless tracking software (TEMA Image Systems) to determine the average rotational speed. Figure 5 shows the average rotational speed of the leg as a function of the driving frequency of the coil system. The leg rotation matches the actuation frequency up to $150 \mathrm{~Hz}$. At higher frequencies, the rotation of the leg is no longer in sync with the rotating field. Higher rotation frequencies can be achieved with a stronger magnetic field or by reducing the friction in the joint, though neither is explored here. A lumped parameter approximation of the viscous damping can be estimated from [11],

$$
\omega_{S-O}=\frac{\tau}{c}
$$

where $\omega_{S-O}$ is the step-out frequency when the magnets are no longer in sync, $\tau$ is the maximum applied torque, calculated from eq. 1 , and $c$ is the viscous damping. The viscous damping in the system was calculated as $13.7 \mu \mathrm{N}-\mu \mathrm{m}-\mathrm{s} / \mathrm{rad}$.

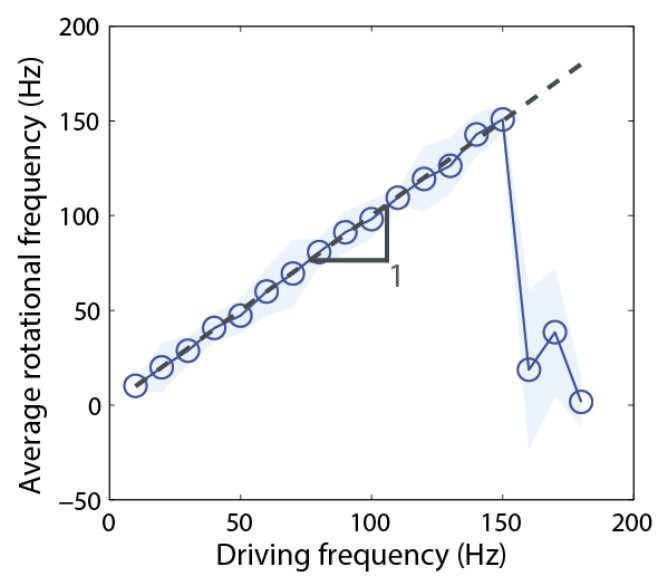

Figure 5: The average rotational frequency of a leg of the microrobot not in contact with the ground vs rotating magnetic field frequency. The solid line represents the average and the shaded area represents the standard deviation over more than five rotations. The dashed line visualizes when the rotational frequency of the leg is equal to the driving frequency.

\section{Wear}

The leg was actuated at $100 \mathrm{~Hz}$ for three hours, corresponding to over $1,000,000$ cycles. A high-speed video taken at $2,500 \mathrm{fps}$ was recorded at 30-minute intervals, corresponding to 180,000 cycle intervals. The data was processed in TEMA to determine the average rotational speed of the mechanism at each video capture. Figure 6 plots the average rotational speed at each 30-minute interval with the shaded area corresponding to one standard deviation. Overall, the average performance does not decrease over $1,000,000$ cycles. However, the relatively large, approximately 15 $\mathrm{Hz}$, standard deviations are a combination of both tracking error and frictional properties in the joint, though no visible signs of wear were observed.

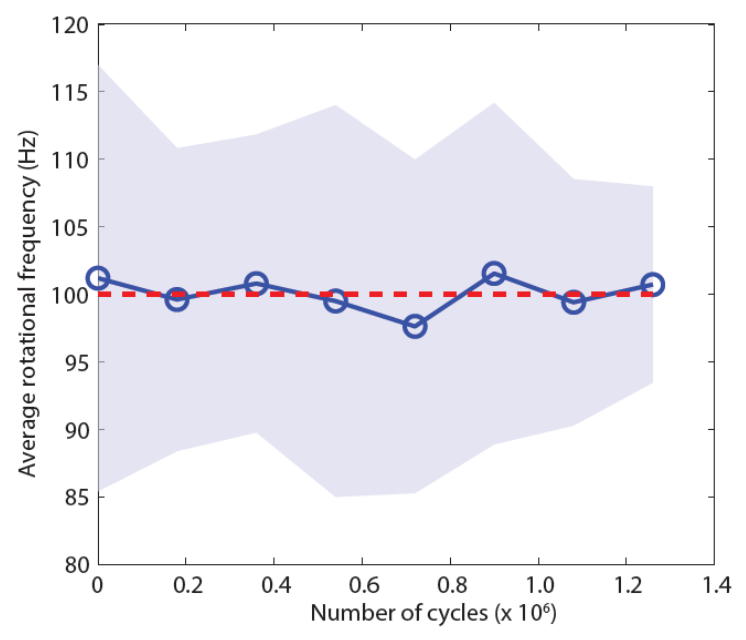

Figure 6: The average rotational frequency (solid line) and standard deviation (shaded area) over ten rotations of the leg actuated at $100 \mathrm{~Hz}$ (dashed red line) captured at 30 minute intervals (180,000 cycle intervals).

\section{LOCOMOTION RESULTS}

The average horizontal velocity of the robot is shown in Figure 7. The solid line corresponds to the average value, while the shaded area represents one standard deviation over 3 trials for one robot. The maximum observed velocity of $37.3 \mathrm{~mm} / \mathrm{s}$ (14.9 body lengths per second) occurs at an actuation frequency $35 \mathrm{~Hz}$. Video stills of the robot running at $23.8 \mathrm{~mm} / \mathrm{s}$ ( 9.52 body lengths per second) are shown in Figure 8.

The average velocity is approximately linear from $5 \mathrm{~Hz}$ to 30 $\mathrm{Hz}$, suggesting that this portion of locomotion is governed largely by the kinematics of the robot design; as frequency increases, horizontal velocity increases proportionally. The slope of this linear fit corresponds to the stride length and is calculated as 0.88 $\mathrm{mm}$, or 0.35 body lengths. In this kinematic dominated regime, changing the effective length of the legs in the robot would provide a longer stride length, and ultimately faster locomotion.

As stride frequency is increased beyond $35 \mathrm{~Hz}$, the velocity performance begins to drop, and becomes more inconsistent with larger variation between trials. In this regime, the robot locomotion is more dynamic, as opposed to kinematic; however, the body dynamics are unfavorable for high-speed running. The robot body was perpetually pitched downward, and the robot propels itself forward with the back legs, rather than performing the pronking gait. During this downward pitched posture, the front of the robot body maintains contact with the ground, reducing the speed of the robot.

At this size scale, surface forces often dominate inertial forces. The Reynolds number $(R e)$ describes a ratio of surface forces to inertial forces and is calculated by

$$
R e=\frac{\rho v D}{\mu},
$$

where $v$ is the velocity of the robot, $D$ is the characteristic length, in this case, body length, and $\mu$ is the dynamic viscosity of air $\left(1.18 \times 10^{-5} \mathrm{~kg} \mathrm{~m}^{-1} \mathrm{~s}^{-1}\right)$, and $\rho$ is the density of air $\left(1.23 \mathrm{~kg} \mathrm{~m}^{-3}\right)$. At the top speed, the Reynolds number is 9.71, signifying that the robot is operating under laminar conditions. Using Stoke's law, an estimate of the drag force is calculated by

$$
F_{D}=\frac{1}{2} \rho v^{2} A C_{D}
$$


where $A$ is the cross-sectional area of the robot $\left(0.98 \mathrm{~mm}^{2}\right)$, and $C_{D}$ is the drag coefficient $\left(C_{D}=2.1\right.$ for a rectangular box $)$. The drag force is equal to $1.77 \mathrm{nN}(0.02 \%$ of the body weight $)$ on the robot running at top speeds. Viscous forces from drag are not dominating the locomotion of the robot, suggesting that inertial forces still play a role in locomotion at the milligram-scale.

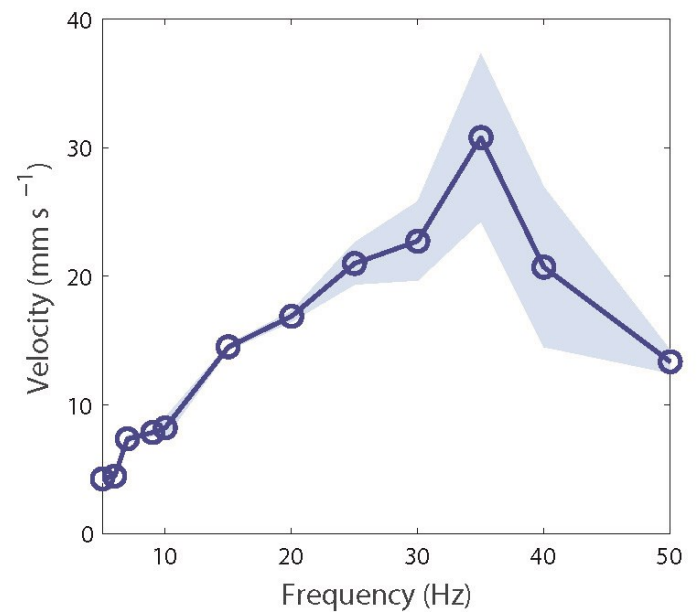

Figure 7: Robot speed vs rotating magnetic field frequency. Solid line represents the average over three trials and the shaded area represents the standard deviation.

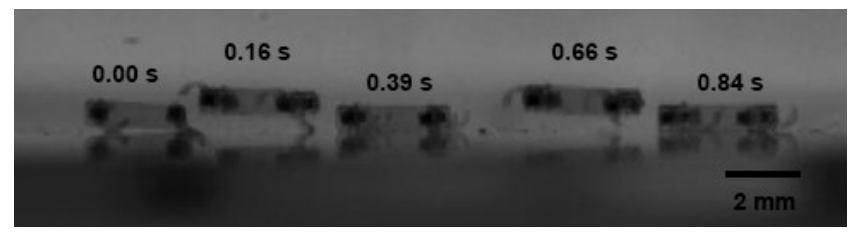

Figure 8: Overlaid frames captured from video of the microrobot walking at approximately 9.5 body lengths per second.

\section{CONCLUSIONS}

This work shows one of the smallest legged robot, capable of achieving biologically relevant speeds, up to 15 body lengths per second. This microrobot also provides a platform for studying locomotion at the milligram scale by incorporating microscale 3D printing with magnetic actuation. The monolithically printed chassis creates friction bearings used in the microrobot to provide full rotary actuation, up to $150 \mathrm{~Hz}$, in this experimental setup, with no signs of visible wear or decreased performance after over $1,000,000$ cycles of actuation.

This work provides an experimental platform to understand the dynamics of locomotion at the milligram-scale. Integrating magnetic actuation provides a sense of the required power and torque needed to achieve movement. Using the calculated maximum torque, the mechanical power of locomotion $(\mathrm{P}=\tau \omega$ $\mathrm{n}_{\text {legs }}$ ) of the robot running at $35 \mathrm{~Hz}$ is $12.1 \mu \mathrm{W}$. Initial insights into the mechanical aspects of legged locomotion show that inertial forces still contribute to locomotion even at the milligram-scale.

\section{ACKNOWLEDGEMENTS}

This work was supported by NSF PECASE Award ECCS1055675.

\section{REFERENCES}

[1] S. Rubin, M. H.-Y. Young, J. C. Wright, D. L. Whitaker, and A. N. Ahn, "Exceptional running and turning performance in a mite," Journal of Experimental Biology, vol. 219, no. 5, pp. 676-685, 2016.
[2] U. Saranli, M. Buehler, and D. E. Koditschek, "Rhex: A simple and highly mobile hexapod robot," International Journal of Robotics Research, vol. 20, no. 7, pp. 616-631, 2001.

[3] T. Ebefors, J. U. Mattsson, E. Kälvesten, and G. Stemme, “A walking silicon micro-robot," in Proceedings of the International Conference on Solid State Sensors and Actuators, pp. 1202-1205, 1999.

[4] S. Hollar, A. Flynn, C. Bellew, and K. Pister, ISolar powered $10 \mathrm{mg}$ silicon robot," in International Conference on Micro Electro Mechanical Systems, pp. 706-711, 2003.

[5] D. S. Contreras, D. S. Drew, and K. S. Pister, "First steps of a millimeter-scale walking silicon robot," in Proceedings of the International Conference on Solid State Sensors and Actuators, pp.910-913, IEEE, 2017.

[6] J. H. Ryou and K. R. Oldham, "Dynamic characterization of contact interactions of micro-robotic leg structures," Smart Materials and Structures, vol. 23, no. 5, p. 055014, 2014.

[7] J. Qu, C. B. Teeple, and K. Oldham, "Modeling legged micro-robot loco- motion based on contact dynamics and vibration in multiple modes and axes,"Journal of Vibrationand Acoustics, 2017.

[8] D. Vogtmann, R. St. Pierre, and S. Bergbreiter, "A 25 mg magnetically actuated microrobot walking at; 5 body lengths/sec," in Proceedings of the International Conference on Micro Electro Mechanical Systems, pp. 179- 182, IEEE, 2017.

[9] R. St. Pierre and S. Bergbreiter, "Gait Exploration of Sub-2 g Robots Using Magnetic Actuation," IEEE Robotics and Automation Letters, vol. 2, pp. 34-40, Jan. 2017.

[10] A. W. Mahoney and J. J. Abbott, "Generating Rotating Magnetic Fields With a Single Permanent Magnet for Propulsion of Untethered Magnetic Devices in a Lumen," IEEE Transactions on Robotics, vol. 30, pp. 411-420, 2014.

[11] N. Ghalichechian, A. Modafe, M. I. Beyaz, and R. Ghodssi, "Design, fabrication, and characterization of a rotary micromotor supported on microball bearings," Journal of Microelectromechanical Systems, vol. 17, no. 3, pp. 632-642, 2008.

[12] L. G. Fréchette, S. A. Jacobson, K. S. Breuer, F. F. Ehrich, R. Ghodssi, R. Khanna, C. W. Wong, X. Zhang, M. A. Schmidt, and A. H. Epstein, "High-speed microfabricated silicon turbomachinery and fluid film bearings," Journal of Microelectromechanical Systems, vol. 14, no. 1, pp. 141- 152, 2005.

[13] S. Kumar, D. Cho, and W. N. Carr, "Experimental study of electric suspension for microbearings," Journal of MicroelectromechanicalSystems, vol. 1, no. 1, pp. 23-30, 1992.

[14] X.-S. Wu, W.-Y. Chen, X.-L. Zhao, and W.-P. Zhang, "Micromotor with electromagnetically levitated rotor using separated coils," Electronics Letters, vol. 40, no. 16, pp. 996$997,2004$.

[15] A. Takei, N. Binh-Khiem, E. Iwase, K. Matsumoto, and I. Shimoyama, "Liquid motor driven by electrowetting," in Proceedings of the International Conference on Micro Electro Mechanical Systems, pp. 42-45, 2008.

[16] M. L. Chan, B. Yoxall, H. Park, Z. Kang, I. Izyumin, J. Chou, M. M. Megens, M. C. Wu, B. E. Boser, and D. A. Horsley, "Design and characterization of MEMS micromotor supported on low friction liquid bearing," Sensors and Actuators A: Physical, vol. 177, pp. 1-9, 2012.

\section{CONTACT}

*R. St. Pierre; rstpierr@umd.edu 